\title{
Path Planning under Kinematic Constraints by Rapidly Exploring Manifolds
}

\author{
Léonard Jaillet and Josep M. Porta
}

\begin{abstract}
The situation arising in path planning under kinematic constraints, where the valid configurations define a manifold embedded in the joint ambient space, can be seen as a limit case of the well-known narrow corridor problem. With kinematic constraints the probability of obtaining a valid configuration by sampling in the joint ambient space is not low but null, which complicates the direct application of sampling-based path planners. This paper presents the AtlasRRT algorithm, a planner specially tailored for such constrained systems that builds on recently developed tools for higher-dimensional continuation. These tools provide procedures to define charts that locally parametrize a manifold and to coordinate the charts forming an atlas that fully covers it. AtlasRRT simultaneously builds an atlas and a bi-directional Rapidly-Exploring Random Tree (RRT), using the atlas to sample configurations and to grow the branches of the RRTs, and the RRTs to devise directions of expansion for the atlas. The efficiency of AtlasRRT is evaluated in several benchmarks involving high-dimensional manifolds embedded in large ambient spaces. The results show that the combined use of the atlas and the RRTs produces a more rapid exploration of the configuration space manifolds than existing approaches.
\end{abstract}

Index Terms-Path Planning, Kinematic Constraints, Manifolds, Higher-Dimensional Continuation

\section{INTRODUCTION}

$\mathbf{T}$ he problem of path planning, i.e., to determine how to move a robotic system from an initial to a goal state avoiding collisions, is ubiquitous in Robotics as it appears in most of the addressed tasks [1, 2]. Sampling-based path planners [3, 4] have been largely successful and are the standard for industry-level solutions [5]. They rely on the fact that while representing the obstacles in configuration space is hard, checking whether a particular configuration is in collision or not is relatively simple. However, these planners have difficulties in the so called narrow corridor problems, where the solution must necessarily traverse a tiny area, i.e., an area with relatively low probability of being sampled. The case where the problem includes kinematic constraints can be seen as a limit case of the narrow corridor problem. In this case, the constraints define a configuration space that is a nullmeasure manifold embedded in the ambient space formed by the robot joint variables [6]. Thus, the probability of directly sampling on the configuration space by selecting random joint values is not just low, but null, which complicates the use of sampling-based path planners.

This work has been partially supported by the Spanish Ministry of Economy and Competitiveness under project DPI2010-18449. Léonard Jaillet was supported by CSIC under a JAE-Doc fellowship partially founded by the ESF

The authors are with the Institut de Robòtica i Informàtica Industrial, CSIC-UPC, Llorens Artigas 4-6, Barcelona 08028, Spain (email: ljaillet@iri.upc.edu; porta@iri.upc.edu).

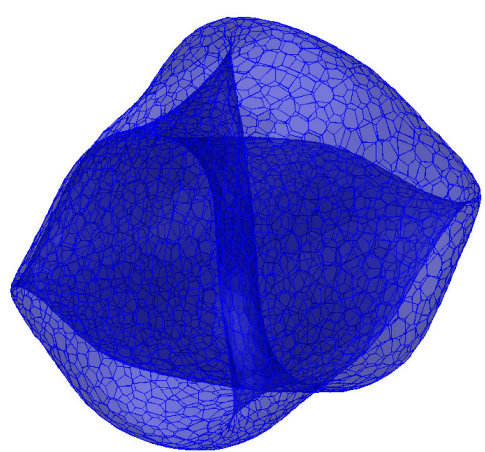

(a)

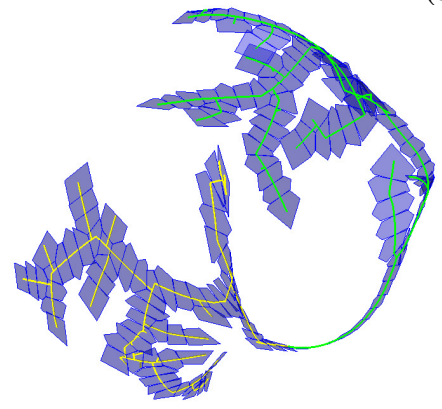

(b)

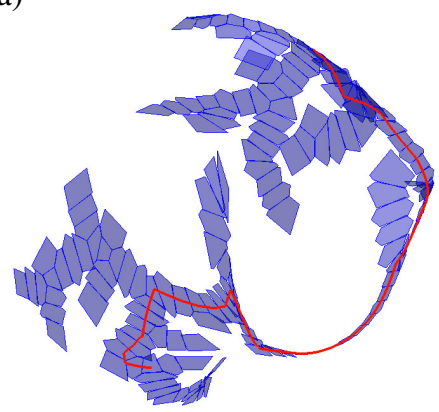

(c)
Fig. 1. Example of exploration with AtlasRRT. (a) Full atlas of the bidimensional configuration space of the cyclooctane. (b) AtlasRRT intertwines the construction of a bidirectional RRT with an atlas construction. The trees rooted at the start and goal configurations are represented in yellow and in green, respectively. (c) When the two RRTs are connected, a solution path (represented in red) can be readily computed. Observe that only a small fraction of the full atlas is necessary to connect the query configurations.

Path planning under kinematic constraints is a classical topic in Robotics [7]-[10] and it appears, for instance, in complex manipulation problems [11], parallel robots [12], robot grasping [13], constraint-based object positioning [14], or surgery robots [15]. This problem is also crucial in Biochemistry, when analyzing the conformational changes in molecular loops [16]. Moreover, the popularization of robots such as two-armed service robots [17], anthropomorphic hands [18], or humanoid robots [19] where loop closures appear very often has strongly pushed the research in this field during the last years [20]-[25]. In the quest of a general and efficient solution, most of the existing approaches try to adapt the sampling-based planners to the constrained case.

The efficiency of the sampling-based path planning approaches relies on the ability of drawing samples and of growing branches that cover the configuration space. If some information about the environment is available, the distribution 
of samples can be adapted to the problem [26]. However, in the absence of such information, a uniform distribution is preferred. This distribution of samples can only be easily obtained using an isometric parametrization of the configuration space. Moreover, the branch extension is typically based on linear interpolation between samples, which also relies on a parametrization of the configuration space. Whereas for nonconstrained systems such parametrization is straightforward, this is not the case when the kinematic constraints reduce the dimensionality of the configuration space. Due to this issue, existing attempts to adapt the sampling-based planners to problems with kinematic constraints are either limited to particular families of mechanisms or unable to efficiently explore the configuration space.

To address these limitations, we propose here a method called AtlasRRT based on a coordinated construction of an atlas and a bidirectional RRT. On the one hand, the atlas is used to adequately sample configurations and grow branches on the configuration space manifold and thus, to retain the RRT exploration efficiency, despite working on a non-Euclidean configuration space. On the other hand, the RRT is used to determine directions of expansion for the atlas, so that the charts generated are those useful to find solution paths. An example of problem solved with AtlasRRT is shown in Fig. 1 that represents the two-dimensional configuration space of the cyclooctane molecule, a bi-directional RRT, and the solution path obtained with the proposed approach. AtlasRRT is an evolution of the algorithm introduced in [27]. Here, the change of the underlying formulation, the improvements in the coordination between the RRT and the atlas, and the new strategies to control the growth of the trees result in a significant speed up with respect to the preliminary version of the algorithm. Moreover, small modifications are introduced in the algorithm to guarantee its probabilistic completeness.

This paper is organized as follows. Next section frames the proposed planner in the context of the previous work. Then, Section III introduces the concepts of charts and atlas and how to use them to represent an implicitly-defined manifold. Section IV presents a way to integrate the atlas-based manifold representation with an RRT exploration. Section V formally describes the algorithms implementing the AtlasRRT planner and Section VI compares its performance to state of the art methods for several benchmarks involving high-dimensional manifolds embedded in large ambient spaces. Finally, Section VII summarizes the contributions and limitations of this work and indicates points that deserve further attention.

\section{RELATED WORK}

As already mentioned, the main issue for path planning under kinematic constraints is to devise proper ways to sample the configuration space manifold and to connect the samples between them.

For some particular families of mechanism with kinematic loops, distance-based formulations provide a global parametrization that can be readily used to sample and to grow branches in the configuration space [23, 28]. Other approaches try to learn the parametrization from large sets of samples
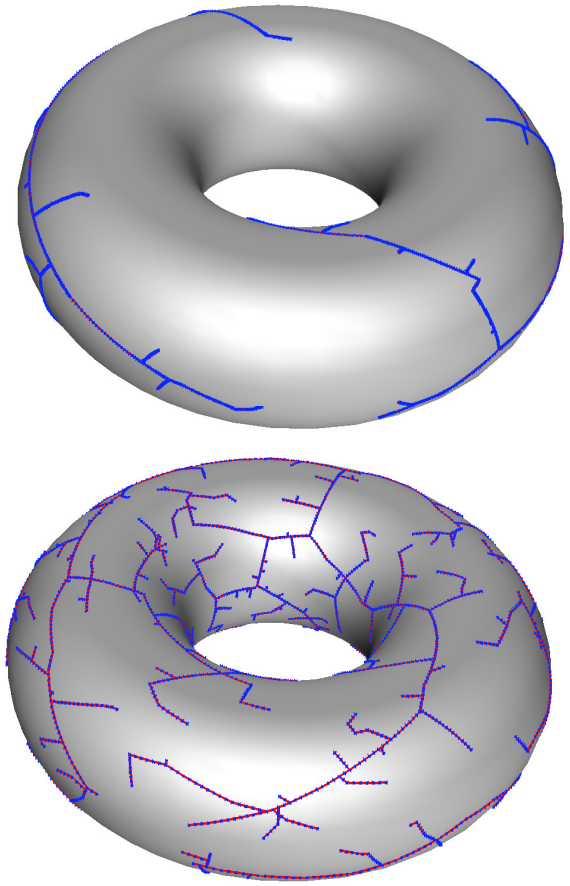

Fig. 2. Two RRTs of 500 samples built on a torus-like manifold. Top With an ambient space sampling method, the exploration focuses on the outer parts of the torus and many samples do not produce any tree extension. Bottom With an AtlasRRT, the diffusion process is largely independent of the ambient space which improves the coverage.

on the configuration space [22], but they can only deal with relatively simple manifolds.

In the absence of a global parametrization, KinematicsPRM [8] samples a subset of joint variables and uses inverse kinematics to find values for the remaining ones. Unfortunately, this strategy is not valid for all the mechanisms, and although some improvements have been proposed [29], the probability of generating invalid samples is significant. Finally, the non uniqueness of the solutions for the inverse kinematic functions and the presence of singularities complicate the approach [30]. Task-space planners [24, 31, 32] are similar in the sense that they sample on a subset of variables (those related with the end-effector), although they typically determine values for the remaining degrees of freedom using numerical techniques instead of closed kinematic functions. Thus, they share the problems of kinematic-based approaches regarding the multiple solutions for the non-fixed variables.

An alternative strategy to get valid configurations is to sample in the joint ambient space and to converge to the configuration space either implementing random walks [9], or the more efficient Jacobian pseudoinverse method [20, 21, 33]. These approaches are probabilistically complete [34] and easy to implement, but a uniform distribution of samples in the ambient space does not necessarily translate to a uniform distribution in the configuration space [24], and the branch extensions are many times prematurely blocked, as noted in [35], which reduces their efficiency. This problem is illustrated in Fig. 2 where the configuration space to be explored is a toruslike manifold of diameter four times smaller than the ambient 
space width. Fig. 2-top shows a RRT built from points sampled in the ambient space that has a poor coverage of the manifold. With the AtlasRRT presented in this paper, the process of diffusion is largely independent of the configuration space shape and of the ambient space bounds which improves the coverage of the manifold, as shown in Fig. 2-bottom.

To increase the quality of the exploration, one can focus on a subset of the ambient space around the configuration space [36]. With this method, though, points are still sampled in the ambient space, which can be of much higher dimensionality than the configuration space. Suh et al [35] introduce a lazy RRT scheme where loosely coordinated RRTs are built on tangent spaces that locally approximate the manifold and that have the same dimensionality as the configuration space. However, the quality of the resulting RRT is affected by the possible overlap of tree branches that belong to different tangent spaces.

From Differential Geometry, it is well known that a manifold can be described by a collection of local parametrizations called charts, that can be coordinated within an atlas [37]. Higher-dimensional continuation techniques provide principled numerical tools to compute the atlas of an implicitly defined manifold starting from a given point, whereas minimizing the overlap between neighboring charts [38, 39]. One-dimensional continuation methods, have been strongly developed in the context of Dynamical Systems [40], whereas in Robotics, they have been mainly used for solving problems related to Kinematics [41, 42]. To the best of our knowledge, higher-dimensional continuation methods have been only used in Robotics to evaluate the dexterity of mechanisms [43, 44]. In a previous work [25], we introduced a resolution complete path planner on manifolds based on higher-dimensional continuation tools. Despite its efficiency, this planner relies on a discretization of the manifold and the exploration could be blocked in the presence of narrow corridors, unless using a fine enough resolution, with the consequent loose in performance. Moreover, the number of charts generated with this planner scales exponentially with the dimension of the configuration space, hindering its application to complex problems. In [27], we preliminarily explored the possibility to combine the atlas construction with an RRT. An improved strategy with stronger theoretical background is presented here.

\section{REPRESENTING IMPLICITLY-DEFINED CONFIGURATIONS SPACES}

Let's consider a system described by a $n$-dimensional joint ambient space $\mathcal{A}$ and a $k$-dimensional configuration space $\mathcal{X} \subset \mathcal{A}$ implicitly defined by a set of equality constraints

$$
\mathcal{X}=\{\mathbf{x} \in \mathcal{A} \mid \mathbf{F}(\mathbf{x})=\mathbf{0}\}
$$

with $\mathbf{F}: \mathcal{A} \rightarrow \mathbb{R}^{n-k}$, and $n>k>0$. We adopt here the convention where the configuration space $\mathcal{X}$ is defined as the set of points fulfilling the constraints [6] (this is sometimes called constrained configuration space) that is embedded in the ambient space of the joint variables (called configuration space in some approaches). The constraints defining the configuration space may either come from the mechanics of

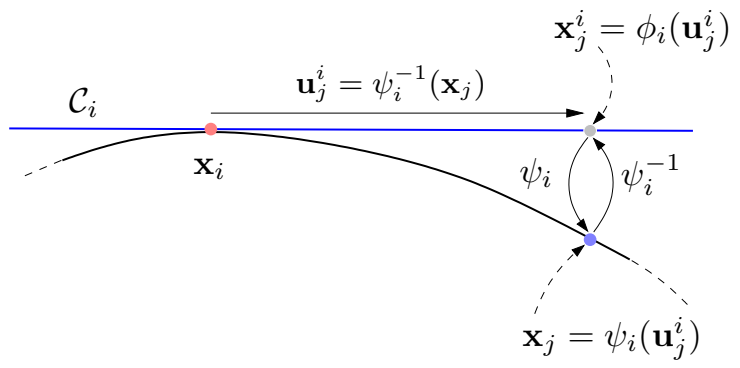

Fig. 3. In this paper, a chart $\mathcal{C}_{i}$ defines an exponential map $\mathbf{x}_{j}=\psi_{i}\left(\mathbf{u}_{j}^{i}\right)$ between the tangent space at $\mathbf{x}_{i}$ and the manifold, as well as a logarithmic map $\mathbf{u}_{j}^{i}=\psi_{i}^{-1}\left(\mathbf{x}_{j}\right)$ between the manifold and the tangent space. The mapping $\phi_{i}$ provides the ambient space coordinates of the tangent space parameters $\mathbf{u}_{j}^{i}$.

the system itself (e.g. the assembly restrictions of a parallel robot) or from the task to be performed (e.g. a tray that must remain horizontal during a given task). Note that we only consider kinematic constraints and other constraints such as those involving dynamical aspects would not be not taken into account. Moreover, we assume that $\mathcal{X}$ is a smooth manifold everywhere, without considering the presence of singularities.

Let $\mathcal{O}$ be the obstacle region of the manifold, such that $\mathcal{F}=\mathcal{X} \backslash \mathcal{O}$ is the open set of the non-colliding configurations. Let also assume that $\mathbf{x}_{s}$ and $\mathbf{x}_{g}$ are the given start and goal configurations respectively, both in $\mathcal{F}$. Then, the path planning problem consists of finding a collision free path linking the query configurations while staying on the manifold i.e. to find a continuous function $\sigma:[0,1] \rightarrow \mathcal{X}$ with $\sigma(0)=\mathbf{x}_{s}$, $\sigma(1)=\mathbf{x}_{g}$, and with $\sigma(\tau) \in \mathcal{F}$ for each $\tau \in[0,1]$.

Representing an implicitly-defined manifold with a global isometric parametrization is infeasible even for simple manifolds such as a sphere in 3D. However, we can represent it as a set of local parametrizations, called charts, that form an atlas that covers the manifold.

Formally, a chart, $\mathcal{C}_{i}$, locally parametrizes the $k$-dimensional manifold around a given point $\mathbf{x}_{i}$ with a bijective map, $\mathbf{x}_{j}=\psi_{i}\left(\mathbf{u}_{j}^{i}\right)$, between parameters $\mathbf{u}_{j}^{i} \in \mathbb{R}^{k}$ and points $\mathbf{x}_{j}$ on the manifold $\mathcal{X}$, with $\psi_{i}(\mathbf{0})=\mathbf{x}_{i}$. The map from the parameter space to the manifold is known as the exponential map and the inverse is referred as the logarithmic map (see Fig. 3). Following [38], an approximation of the exponential and logarithmic maps can be implemented using the $k$-dimensional space tangent at $\mathbf{x}_{i}$. An orthonormal basis for this tangent space is given by the $n \times k$ matrix, $\boldsymbol{\Phi}_{i}$, satisfying

$$
\left[\begin{array}{c}
\mathbf{J}\left(\mathbf{x}_{i}\right) \\
\mathbf{\Phi}_{i}^{\top}
\end{array}\right] \boldsymbol{\Phi}_{i}=\left[\begin{array}{l}
\mathbf{0} \\
\mathbf{I}
\end{array}\right],
$$

with $\mathbf{J}\left(\mathbf{x}_{i}\right)$ the Jacobian of $\mathbf{F}$ evaluated at $\mathbf{x}_{i}$, and $\mathbf{I}$, the $k \times k$ identity matrix. Using this basis, the exponential map $\psi_{i}$ is determined by first computing the mapping $\phi_{i}$ from parameters in the tangent space to coordinates in the joint ambient space,

$$
\mathbf{x}_{j}^{i}=\phi_{i}\left(\mathbf{u}_{j}^{i}\right)=\mathbf{x}_{i}+\boldsymbol{\Phi}_{i} \mathbf{u}_{j}^{i},
$$

and then, orthogonally projecting this point on the manifold to obtain $\mathbf{x}_{j}$. This projection can be computed by solving the system

$$
\left\{\begin{aligned}
\mathbf{F}\left(\mathbf{x}_{j}\right) & =\mathbf{0} \\
\mathbf{\Phi}_{i}^{\top}\left(\mathbf{x}_{j}-\mathbf{x}_{j}^{i}\right) & =\mathbf{0},
\end{aligned}\right.
$$




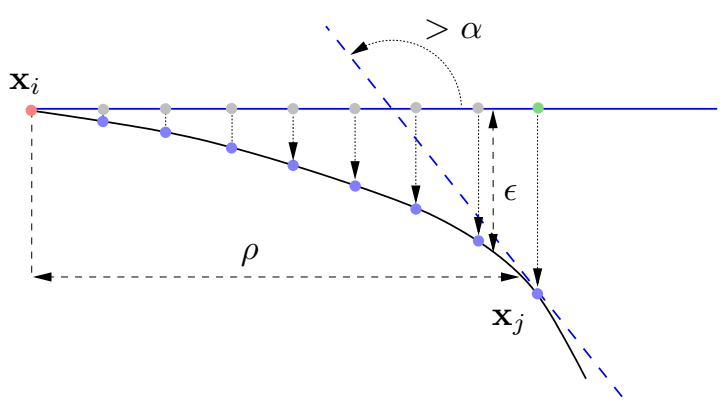

Fig. 4. Validity area of a chart $\mathcal{V}_{i}$ defined from Eqs. (7) to (9). To ensure a correct mapping between the chart and the manifold, the distance and the curvature with respect to the tangent space must be bounded. Moreover, $\mathcal{V}_{i}$ is bounded to cover an area of maximum radius $\rho$ to obtain a regular paving of the manifold. In the figure, the green point is not part of $\mathcal{V}_{i}$ since it does not fulfill these conditions.

using a Newton procedure where $\mathbf{x}_{j}$ is initialized by an approximation of the solution $\left(\mathbf{x}_{j}^{i}\right.$ if no better approximation is available) and is iteratively updated by the $\Delta \mathbf{x}_{j}$ increments fulfilling

$$
\left[\begin{array}{c}
\mathbf{J}\left(\mathbf{x}_{j}\right) \\
\boldsymbol{\Phi}_{i}^{\top}
\end{array}\right] \Delta \mathbf{x}_{j}=-\left[\begin{array}{c}
\mathbf{F}\left(\mathbf{x}_{j}\right) \\
\mathbf{\Phi}_{i}^{\top}\left(\mathbf{x}_{j}-\mathbf{x}_{j}^{i}\right)
\end{array}\right]
$$

until the error is negligible or for a maximum number of iterations [45].

The logarithmic mapping $\psi_{i}^{-1}$ can be computed as the projection of a point on the tangent space

$$
\mathbf{u}_{j}^{i}=\psi_{i}^{-1}\left(\mathbf{x}_{j}\right)=\boldsymbol{\Phi}_{i}^{\top}\left(\mathbf{x}_{j}-\mathbf{x}_{i}\right) .
$$

Note that this projection can be also applied to points not on the manifold to obtain their parameters in a given tangent space.

The area of the manifold properly parametrized by a given chart is limited. As the norm of $\mathbf{u}_{j}^{i}$ increases, the distance to the manifold and the difference in curvature typically increase too, and the Newton process implementing $\psi_{i}$ could even diverge. Thus, the validity area $\mathcal{V}_{i}$ of chart $\mathcal{C}_{i}$ is defined as the set of points $\mathbf{u}_{j}^{i}$ such that $\psi_{i}$ can be safely computed and where

$$
\begin{aligned}
\left\|\mathbf{x}_{j}-\phi_{i}\left(\mathbf{u}_{j}^{i}\right)\right\| & \leq \epsilon, \\
\left\|\boldsymbol{\Phi}_{i}^{\top} \boldsymbol{\Phi}_{j}\right\| & \geq \cos (\alpha), \\
\left\|\mathbf{u}_{j}^{i}\right\| & \leq \rho,
\end{aligned}
$$

with $\boldsymbol{\Phi}_{j}$ the basis of the tangent space at $\mathbf{x}_{j}=\psi_{i}\left(\mathbf{u}_{j}^{i}\right)$. The first condition limits the maximal distance error between the tangent space and the manifold whereas the second condition ensures a bounded curvature in the part of the manifold covered by $\mathcal{C}_{i}$, as well as a smooth transition between charts (see Fig. 4). Combined, these two conditions bound the distorsion introduced by the exponential map. Thus, they ensure that a uniform distribution of samples in $\mathcal{V}_{i}$ translates to an approximately uniform distribution of configurations on the manifold, and that a straight line in tangent space gives a path on the manifold that has a bounded error with respect to the geodesic [46]. Finally, the third condition is introduced to obtain a more regular paving of the manifold.

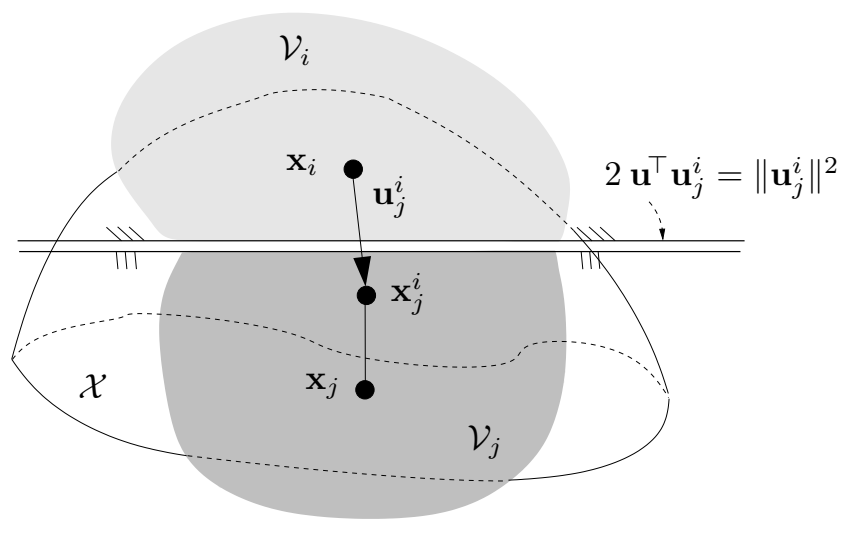

Fig. 5. When a new chart is defined at $\mathbf{x}_{j}$, the validity areas of the two charts, $\mathcal{V}_{i}$ and $\mathcal{V}_{j}$, are coordinated to avoid overlaps.
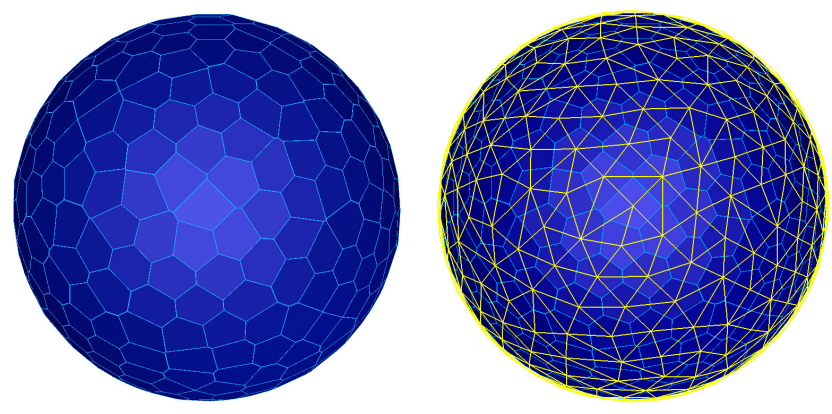

Fig. 6. Left Atlas of a sphere. Each polygonal patch corresponds to a given $\mathcal{P}_{i}$, a conservative approximation of the validity area for the associated chart. Right A roadmap can be extracted from the atlas where the nodes are the chart centers and the edges are given by the neighborhood relations between charts. This roadmap could be used to devise collision free paths between any two given configurations.

The applicability area of a given chart, $\mathcal{V}_{i}$, is never empty and it always includes the center of the chart, $\mathbf{x}_{i}$, but its shape can be arbitrarily intricate. Thus, $\mathcal{V}_{i}$ is conservatively approximated with a convex polytope, $\mathcal{P}_{i} \subset \mathcal{V}_{i}$. This polytope is respresented with a set of linear inequalities, $\mathcal{L}_{i}$, defined in the tangent space associated with the chart [38]. This set is initially empty and is enlarged as the borders of $\mathcal{V}_{i}$ are discovered. If a parameter vector $\mathbf{u}_{j}^{i}$ is in $\mathcal{V}_{i}$ but $\mathbf{u}_{j}^{i}+\Delta \mathbf{u}$ is not in this set for a small $\Delta \mathbf{u}$, then a new chart $\mathcal{C}_{j}$ is added to the atlas at $\mathbf{x}_{j}=\psi_{i}\left(\mathbf{u}_{j}^{i}\right)$ and the following inequality

$$
2 \mathbf{u}^{\top} \mathbf{u}_{j}^{i} \leq\left\|\mathbf{u}_{j}^{i}\right\|^{2},
$$

is included in $\mathcal{L}_{i}$, as shown in Fig. 5. Such inequality reduces the validity regions of the charts by cropping a half space defined in the tangent space associated to $\mathcal{C}_{i}$ given by the plane orthogonally bisecting vector $\mathbf{u}_{j}^{i}$. Actually, the new chart is coordinated not only with $\mathcal{C}_{i}$ but with all the charts $\mathcal{C}_{l}$ in the atlas such that $\mathbf{x}_{j} \in \mathcal{V}_{l}$. Thus, the set of inequalities for $\mathcal{C}_{j}$ is initialized with the inequalities defined from $\mathbf{u}_{l}^{j}=\phi_{j}^{-1}\left(\mathbf{x}_{l}\right)$, the projection of the centers of the nearby charts, $\mathcal{C}_{l}$, on the new chart $\mathcal{C}_{j}$.

Using the exponential and logarithmic maps, a full atlas of the manifold can be defined from where a roadmap whose edges are given by the neighboring relations between charts 
can be extracted (see Fig. 6). In principle one could implement a resolution complete planner using such roadmap to determine an optimal path between any two given configurations. However, this process would be computationally too demanding, specially in high dimensions [25]. Therefore, we propose to intertwine the atlas construction with the definition of a RRT to focus the exploration on the regions of the manifold relevant for the considered path planning problem.

\section{RAPID EXPLORATION OF IMPLICITLY-DEFINED CONFIGURATION SPACES}

Instead of extending the atlas by following a predefined sequence of steps, we propose to use a RRT to devise random directions of expansions for the atlas. This trades off completeness in the exploration by efficiency: the RRT drives the growth of the atlas towards yet unexplored regions of the configuration space, delaying the refinement of already explored areas.

A RRT construction mechanism is based on three basic operations that are described next for the case of implicitlydefined manifolds: sampling in the space to explore, detection of nearest-neighbors, and extension of the tree towards a given point.

\section{A. Random Sampling}

To sample in $\mathcal{X}$ we take advantage of the partially built atlas. First, a chart $\mathcal{C}_{r}$ is randomly selected and then, a point is randomly sampled within the ball of radius $\rho_{s}>\rho$ defined on the tangent space associated with this chart. If the random point is not in $\mathcal{P}_{r}$, the polytope that approximates the validity region of $\mathcal{C}_{r}$, it is discarded. This process is repeated until a valid sample $\mathbf{u}_{r}$ is eventually found. When the sampling is successful, $\mathbf{u}_{r}$ can be translated to coordinates in the ambient space using the corresponding $\phi_{r}$ mapping to get $\mathbf{x}_{r}$. Note that there is no need to map the parameters to $\mathcal{X}$ since the branch extension is actually performed in the tangent space, projecting on $\mathcal{X}$ when necessary.

For a given atlas, the probability of generating a valid random point in chart $\mathcal{C}_{i}$ is proportional to the volume of $\mathcal{P}_{i}$ and therefore, the sampling process selects points uniformly distributed within the region covered by the atlas. Since the atlas is defined incrementally, chart generated at the beginning of the process can be selected more times. These charts, however, typically have a larger rejection ratio than charts at the frontier of exploration, that have sampling areas much larger than their actual validity areas. Therefore, in practice, the exploration is pushed towards non-explored regions of the configuration space. Actually, the ratio $1-\left(\rho / \rho_{s}\right)^{k}$ gives an upper bound of the proportion of sampled points that are outside the validity region of a given frontier chart and, thus, the ratio of points that will trigger the creation of new charts. Therefore, by changing $\rho_{s}$, we can directly tune the balance between exploration and refinement, that is an important feature of the RRT-based algorithms [47].
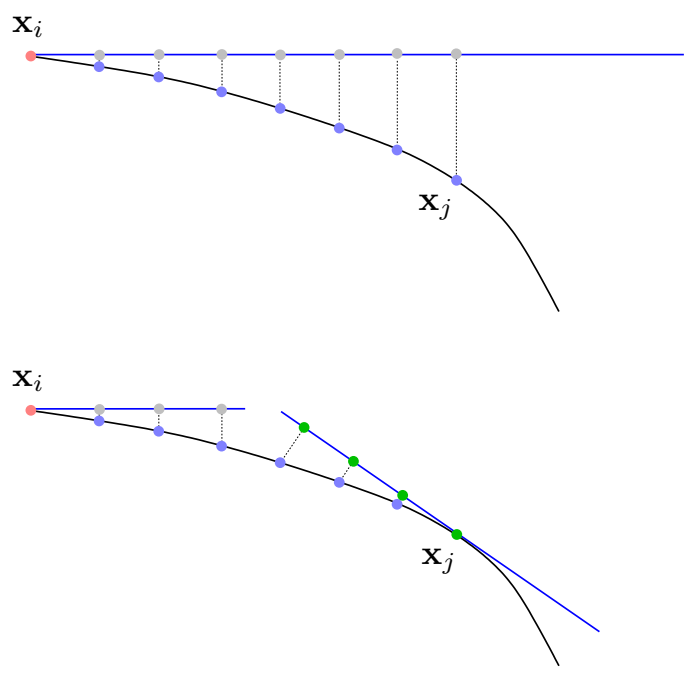

Fig. 7. Top RRT nodes parametrized by the chart at $\mathbf{x}_{i}$. Bottom When a new chart is created at $\mathbf{x}_{j}$, some of the nodes are now parametrized by the new chart. The nodes whose parametrization changes are represented in green.

\section{B. Nearest Neighbor}

The second basic operation to build a RRT is the identification of the node $\mathbf{x}_{n}$ in the tree closer to the random node, $\mathbf{x}_{r}$. This should be done using the intrinsic metric of the configuration space. In a parametrizable space this metric is simple, but on a manifold, the geodesic distance should be used. The implementation of an efficient nearest-neighbor procedure for implicitly-defined manifolds is difficult and it has been only addressed recently in an approximated way relying on a representation of the manifold that is very similar to a partial atlas [48]. However, the approximation is only adequate for dense set of samples, that is not the case when building a RRT. An alternative solution, that will be adopted here, is to resort to the ambient space nearest-neighbor as an approximation of the geodesic nearest-neighbor, despite this may sometimes lead to inadequate tree extensions.

\section{Tree Extension}

When the nearest node, $\mathbf{x}_{n}$, has been selected, the RRT proceeds to grow a branch towards the random sample $\mathbf{x}_{r}$. Note that $\mathbf{x}_{n}$ and $\mathbf{x}_{r}$ can be in different charts and, thus, given in different local parametrizations. Therefore, the direction of extension is obtained by projecting $\mathbf{x}_{r}$ on the chart $\mathcal{C}_{c}$ parametrizing $\mathbf{x}_{n}$ and eventually translating the resulting point to ensure that it is at least at distance $\left\|\mathbf{x}_{n}-\mathbf{x}_{r}\right\|$ from $\mathbf{x}_{n}$. Once the nearest node and the random point are represented in the same parameter space, a new branch can be generated by linear interpolation between the parameters of these two points. After each interpolation step, the parameters are projected on the configuration space and, if the new configuration is collision free, a new node is added to the tree.

During the tree extension the new branch might leave $\mathcal{P}_{c}$ or $\mathcal{V}_{c}$. If it leaves $\mathcal{P}_{c}$, then the branch extension must continue on a neighboring chart. If the branch reaches a yet unknown region that borders $\mathcal{V}_{c}$, a new chart has to be created at the last 


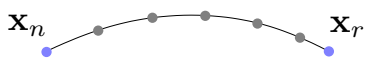

(a)

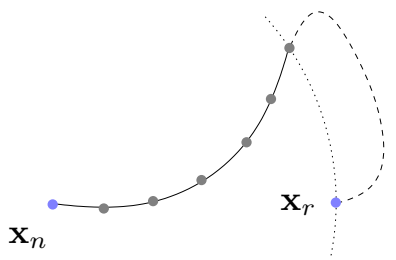

(c)

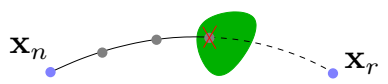

(b)

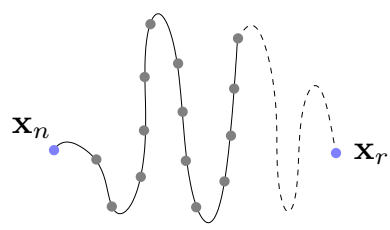

(d)
Fig. 8. The four conditions for stopping the AtlasRRT branch extension process. (a) The branch reaches the random configuration. (b) A collision is detected. (c) The distance from the end branch to its origin is larger than the distance to the random point. (d) The distance traveled is larger than $\lambda$ times the distance to the random point.

point within $\mathcal{V}_{c}$. After creating a chart, the nodes in existing charts not fulfilling the inequalities introduced by the new chart move to the area of validity of the new chart (see Fig 7). Whenever the branch reaches a new or a neighboring chart, the direction of expansion is recomputed, projecting $\mathbf{x}_{r}$ on this chart and ensuring that it is far enough from the last node added to the RRT. This projection strategy is different from the one in [27], where branch extensions were prematurely stopped in some occasions.

The most expensive step in the branch generation is the evaluation of Eq. (8) since it requires to build the tangent space at each considered point. This cost can be alleviated evaluating the curvature along the direction of expansion of the branch. This operation only requires the distance between the previous and the new nodes in parameter and in ambient spaces, which are readily available. Such simplified curvature evaluation is not present in [27] and yields a large execution speed up. Note, however, that the full curvature test is used when a new chart is added to the atlas. Thus, neighboring relations between charts always fulfill Eq. (8).

When exploring an Euclidean space with a RRT, a branch extension stops either when the random sample is reached or when a collision is detected. When exploring a non-Euclidean space, a branch can be arbitrarily long due to the manifold curvature, even when using the two conditions above. To avoid this issue, the branch extension is also stopped if

$$
\left\|\mathbf{x}_{j}-\mathbf{x}_{r}\right\|>\left\|\mathbf{x}_{n}-\mathbf{x}_{r}\right\|
$$

or if

$$
d>\lambda\left\|\mathbf{x}_{n}-\mathbf{x}_{r}\right\|,
$$

with $\mathbf{x}_{j}$ the last node added to the branch, $\mathbf{x}_{r}$ the random sample, $\mathbf{x}_{n}$ the nearest node in the tree, and $d$ the length of the branch, as illustrated in Fig. 8. The first condition prevents the branch to escape the ball centered at $\mathbf{x}_{n}$ with radius $\left\|\mathbf{x}_{n}-\mathbf{x}_{r}\right\|$. The second condition avoids the length of the branch to be larger that a scale factor of the distance between $\mathbf{x}_{r}$ and $\mathbf{x}_{n}$. These two branch termination conditions were not present in the preliminary version of the algorithm [27] and largely help to improve the quality of the RRT.
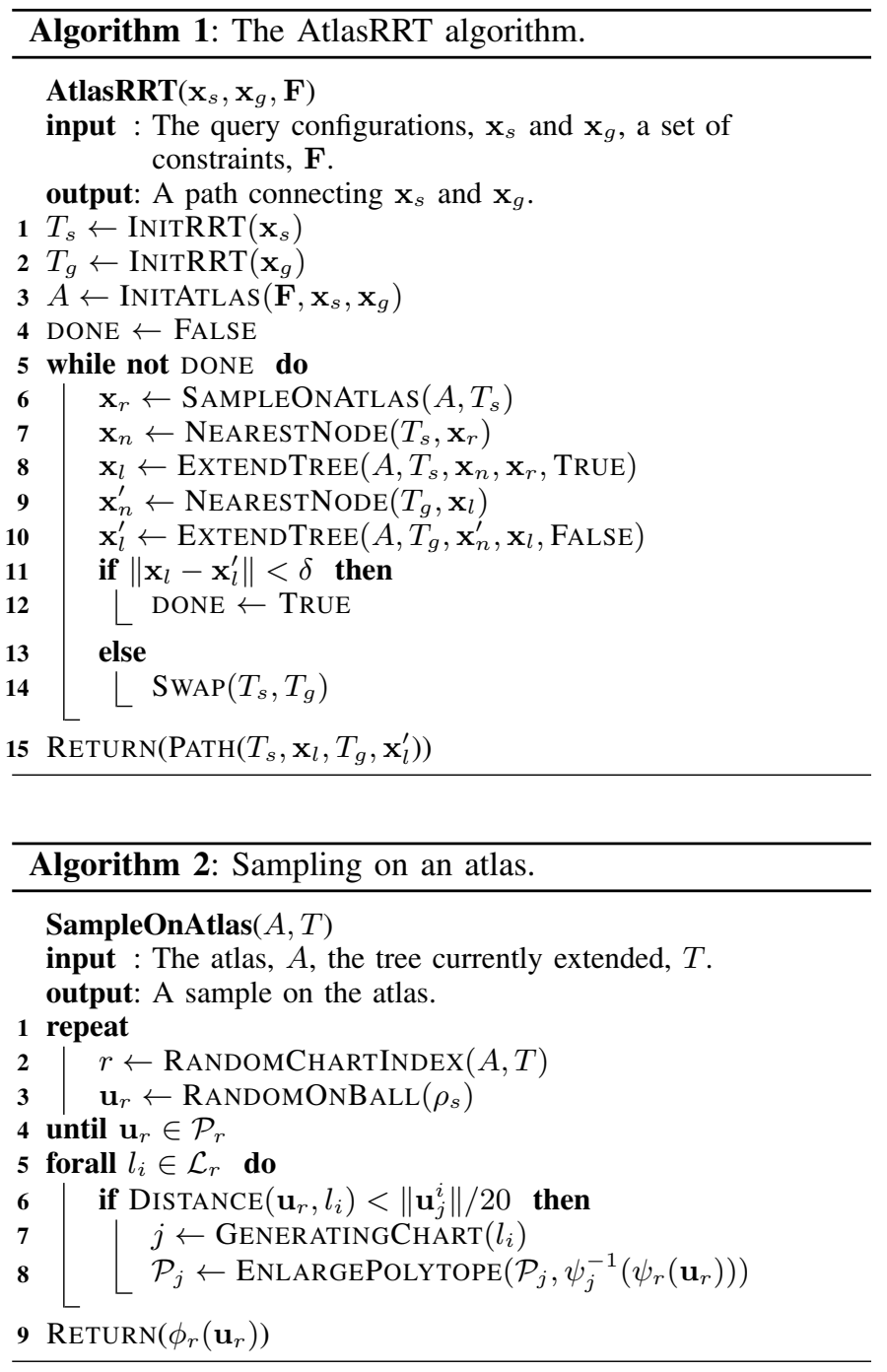

\section{AtLAsRRT Algorithm}

Algorithm 1 gives the pseudo-code for the AtlasRRT planner implementing the path planning approach introduced in the previous section. The algorithm takes $\mathbf{x}_{s}$ and $\mathbf{x}_{g}$ as start and goal configurations, respectively, and tries to connect them with a path on the manifold implicitly defined by a given set of constraints $\mathbf{F}$. The algorithm implements a bidirectional RRT with one tree rooted at $\mathbf{x}_{s}$ and another at $\mathbf{x}_{g}$ (lines 1 and 2). An atlas is also initialized with one chart centered at each one of these points (line 3). Next, the algorithm iterates, trying to connect the two trees (lines 5 to 14). First, a configuration is sampled from the atlas (line 6) and a RRT branch is extended from the nearest node already in the tree (line 7) towards the random sample (line 8). Then, an extension attempts to reach the last node of the new branch (line 10) from the nearest node in the other tree (line 9). If this second extension achieves its objective (line 11), the trees are connected and their nodes are used to reconstruct a path between $\mathbf{x}_{s}$ and $\mathbf{x}_{g}$ (line 15). Otherwise, the two trees are swapped (line 14) and the extension process is repeated.

In the sampling process, we use the atlas as described in Algorithm 2. A chart is selected at random with uniform 


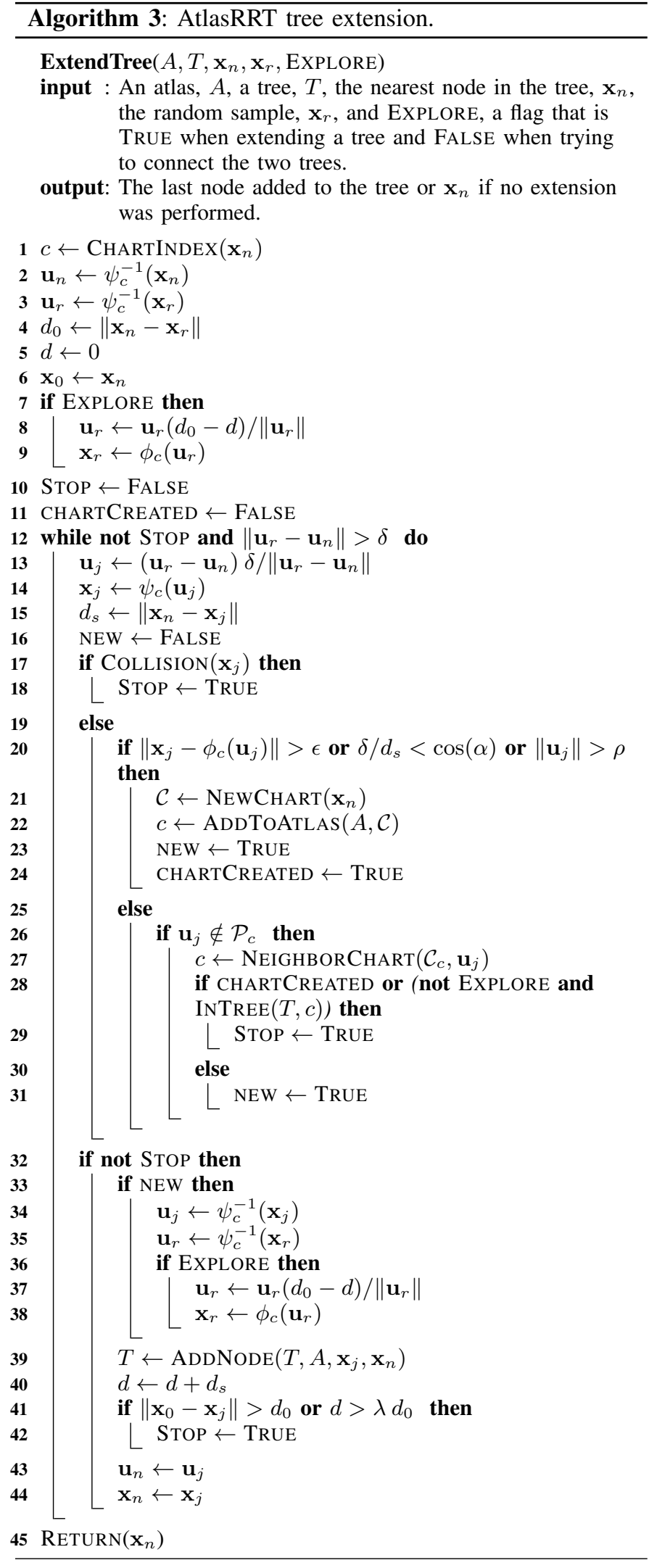

distribution among the set of charts reached by the tree to extend (line 2). Then, a point, $\mathbf{u}_{r}$, is sampled within the ball of radius $\rho_{s}$ bounding the sampling region of this chart (line 3). The process is repeated until a point in $\mathcal{P}_{r}$ is obtained (line 4). Sampling only in the charts reached by the tree being extended produces a more regular exploration than when using all the charts of the atlas [27]. For the samples close to the borders defining $\mathcal{P}_{r}$ (line 6), we check whether or not they are also covered by the corresponding neighboring chart (lines 7 and 8). In this test, $\mathbf{u}_{j}^{i}$ is the point used to generate the linear inequality $l_{i}$ and $\left\|\mathbf{u}_{j}^{i}\right\| / 20$ is a tenth of its distance to the chart center. Such a check ensures a small overlap between neighboring charts and, thus, that all the configurations are effectively covered by the atlas and can be selected as random samples. The parameters of the random sample in the neighboring chart are obtained using the exponential map for the chart selected at random, $\psi_{r}$ and, then, the logarithmic map for the neighboring chart, $\psi_{j}^{-1}$. Finally, the random sample returned by the algorithm is formed by the ambient space coordinates for the selected point computed using the mapping $\phi_{r}$ for the selected chart, $\mathcal{C}_{r}$ (line 9).

The addition of a branch to a tree $T$ is done by following the steps detailed in Algorithm 3. Note that there are two types of tree extensions. The first one attempts to reach a random sample defined on the tangent space associated with a given chart. The second one tries to reach a node in the other tree and, thus, a point on the configuration manifold. In the first case, the parameter EXPLORE is set to TRUE and this results in some differences in how the random sample is managed during the tree expansion. In any case, the procedure operates in the chart $\mathcal{C}_{c}$ including the node to be extended, that is initially the chart parametrizing the nearest node $\mathbf{x}_{n}$ (line 1). The sample from where to start the new branch and the sample to reach in the tree extension are both projected on $\mathcal{C}_{c}$ (lines 2 and 3 ) and the distance $d_{0}$ between these two samples is computed (line 4). Moreover, the length of the new branch, $d$, is initialized to 0 (line 5) and the initial branch point is stored in $\mathbf{x}_{0}$ (line 6). The values for $d_{0}, d$, and $\mathbf{x}_{0}$ are necessary to eventually stop the branch extension. If EXPLORE is TRUE, i.e., if the random sample is not on the configuration manifold, the parameters for the random sample are displaced to a distance $d_{0}$ from $\mathbf{u}_{n}$ (lines 8 and 9) to ensure a minimum branch length (see Fig. 9). Then, the branch is extended while none of the possible stop conditions is detected and the random sample is not reached (lines 12 to 44). At each iteration, a node is added to the tree. To define the node to add, a small step of size $\delta$ is taken in the parameter space of $\mathcal{C}_{c}$ from the current node $\mathbf{u}_{n}$ towards $\mathbf{u}_{r}$ (line 13). The resulting parameters $\mathbf{u}_{j}$ are projected on the manifold to obtain the configuration $\mathbf{x}_{j}$ (line 14). If this new configuration is in collision, the branch extension is stopped (line 18). Otherwise, the algorithm checks if the new configuration triggers the generation of a new chart (line 20). If so, the chart is generated at the previous configuration, that is still in $\mathcal{V}_{c}$ and it is added to the atlas (line 22). Configurations that do not generate a new chart might be out of $\mathcal{P}_{c}$, i.e., they might be in the area of the manifold parametrized by a neighboring chart (line 26). If a branch that triggered the creation of new charts enters a preexisting chart, or if the branch enters a chart already reached by the tree under expansion when aiming to reach the other tree, the branch extension is stopped. These situations are typically produced when a branch is extended too far of 

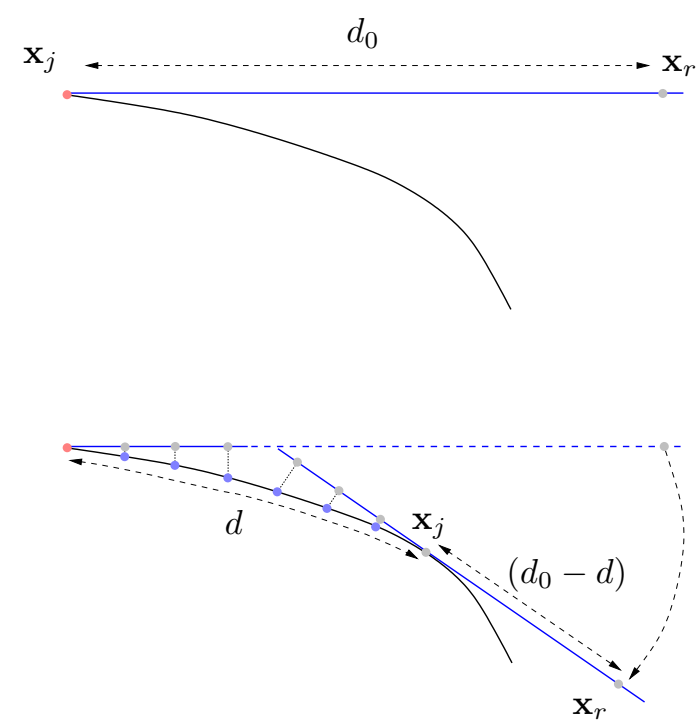

Fig. 9. Top Samples $\mathbf{x}_{r}$ are generated in the tangent space associated with a given chart. Bottom When the RRT branch that attempts to reach $\mathbf{x}_{r}$ generates a chart, the random sample is projected on this new chart and it is displaced to ensure that it is at an adequate distance from the sample from where to continue the branch extension.

when the nearest-neighbor is not actually the closest node to the target sample. By stopping those branch extensions, we avoid an excessive refinement of regions already covered by the tree, which would hinder the efficient exploration of the configuration space manifold. Whenever the branch extension is not stopped, the neighboring chart from where to continue the branch growth is identified (line 27) and the position of the goal sample is recomputed in this new chart (lines 34 to 38). Then, the new node is added to the tree (line 39) and the branch length is updated (line 40). This length is used to determine if the new sample is too far away from the initial branch node or if the branch is too long (line 41). In any of these two cases the branch extension is stopped (line 42). Finally, the new node is set as the point from where to continue the branch extension (lines 43 and 44).

\section{A. Computational complexity}

Besides the cost of collision detection, the most expensive steps in the algorithm are the search for nearest nodes in the RRTs (lines 7 and 9 of Algorithm 1), the search for the neighboring charts when adding a chart to the atlas (line 22 of Algorithm 3), the creation of new charts (line 21 in Algorithm 3), and the computation of the mapping $\psi_{c}$ (line 14 of Algorithm 3). The two search operations can be implemented using hierarchical structures reducing their cost to be logarithmic in the number of nodes of the corresponding RRT and in the number of charts in the atlas, respectively. The cost of generating a new chart is $O\left(n^{3}\right)$ due to the computation of the tangent space basis for which a $\mathrm{QR}$ decomposition is used. Note, though, that the generation of new charts is seldom necessary and, thus, this cost is amortized over several iterations. Finally, the cost of computing the mapping $\psi_{c}$ also scales with $O\left(n^{3}\right)$ since it is implemented as a Newton process with a bounded number of iterations where at each iteration a QR decomposition is used. This Newton process, though, typically converges in very few iterations since it can be initialized at $\mathbf{x}_{n}$, which is very close to the solution point.

\section{B. Parameters}

The algorithm basically uses six parameters: $\epsilon$, the maximum error with respect to a chart used in Eq. (7), $\alpha$, the maximum angle between neighboring charts used in Eq. (8), $\rho$, the maximum radius of the validity area of a chart used in Eq. (9), $\rho_{s}$, the sampling radius, $\delta$, the size branch extension steps, and $\lambda$, the length factor used to eventually stop the branch extensions. Note that both $\delta$ and a bound on the space to sample (the role $\rho_{s}$ in our case) are also used in RRTs in Euclidean spaces.

The parameters $\epsilon, \alpha$, and $\rho$ control the size of the validity area of the charts and, thus, the number of charts in the atlas. Whereas $\epsilon$ and $\rho$ can safely span over a wide range, $\alpha$ should be limited to avoid a large distorsion between the tangent space and the manifold [46]. The ratio between, $\rho$ and $\rho_{s}$ sets the balance between refinement in current charts and exploration since the larger $\rho_{s}$ with respect to $\rho$, the stronger the bias towards unexplored regions. Thus, the ratio $\left(\rho / \rho_{s}\right)^{k}$ should decrease as the dimensionality of the configuration manifold increases, to emulate the exploration bias of RRT in Euclidean spaces. Since no collision nor curvature test is done between two consecutive points in a RRT branch, a small $\delta$ should be used to avoid undetected collision or sharp changes in the manifold curvature. Finally, $\lambda$ must be larger than 1 . However, the performance of the algorithm is not very sensitive to its actual value since the branch termination condition using this parameter is seldom active.

\section{Probabilistic completeness}

To show the probabilistically completeness of the proposed approach, recall that we assumed the configuration space to be a $k$-dimensional smooth manifold, $\mathcal{X}$, with a Jacobian that is full rank everywhere. Under these conditions, $\mathcal{X}$ and its Jacobians are continuous in an open $k$-dimensional ball around any given point on $\mathcal{X}$ and, thus, $\mathcal{X}$ is singularity free and of constant dimensionality. Thus, a path between any two given configurations never includes points on any particular low dimensional subset with null measure. Additionally, by the implicit function theorem, the above conditions also guarantee that the validity area $\mathcal{V}_{i}$ of a given chart centered at $\mathrm{x}_{i}$ includes a non-null measure ball around this point, i.e., $\mathcal{V}_{i}$ is not null in all directions. Moreover, since transitions between different connected components of the manifold are not possible, we assume that the start and the goal configurations are in $\mathcal{F}$, the connected component of the collision free part of $\mathcal{X}$ including both configurations. For simplicity, we consider a version of AtlasRRT where a single tree is generated (the extension of the argument to bidirectional trees is straightforward) and we assume that for each RRT node the curvature test in Eq. (8) is performed, instead of the simplified version described in Section IV-C. In this way, points out of the validity area are detected, independently of the direction from which they 
are approached. Finally, we also assume that the measure of $\mathcal{F}, \mu(\mathcal{F})$, is finite either because its has a closed topology or because it is cropped to some domain, $\mathcal{D}$. Note that this is typically the case in Robotics, where $\mathcal{F}$ is bounded by the joint ranges and the presence of obstacles.

As proved in [34], under the above assumptions, any RRTlike method able to densely sample $\mathcal{F}$ is probabilistically complete, even if the samples are not uniformly distributed. In the AtlasRRT, the sampling is obtained using the atlas. Thus, if we prove that the atlas fully covers $\mathcal{F}$, i.e., that any point on $\mathcal{F}$ is mapped from a point on the atlas, the probabilistic completeness will be guaranteed. To show that an atlas defined using the method presented in Section III fully parametrizes $\mathcal{F}$, we will adapt the argument given in [38]. This argument basically shows that each new chart increases the coverage of $\mathcal{F}$ until it is fully covered.

Let $\mathcal{F}_{A}$ be the part of $\mathcal{F}$ already parametrized by the current atlas $A$, i.e., the projection on $\mathcal{F}$ of the validity areas of all the charts in $A$ where the validity area of chart $\mathcal{C}_{i}$ is

$$
\mathcal{V}_{i}=\mathcal{B}^{k}(\rho) \cap \mathcal{P}_{i}
$$

with $\mathcal{B}^{k}(\rho)$ the $k$-dimensional ball of radius $\rho$ and $\mathcal{P}_{i}$ the polytope associated with the chart. This area is projected on a patch of $\mathcal{F}$ using Eq. (4). Moreover, the sampling area of the chart is

$$
\mathcal{S}_{i}=\mathcal{B}^{k}\left(\rho_{s}\right) \cap \mathcal{P}_{i},
$$

with $\rho_{s}>\rho$. For any open chart, i.e., a chart where $\mathcal{S}_{i} \nsubseteq \mathcal{V}_{i}$, a sample out of $\mathcal{F}_{A}, \mathbf{x}_{r}$, will be generated with probability $\mu\left(\mathcal{S}_{i} \backslash \mathcal{V}_{i}\right)>0$. The RRT branch toward $\mathbf{x}_{r}$ necessarily crosses the border of $\mathcal{F}_{A}$ connecting a RRT node $\mathbf{x}_{n} \in \mathcal{F}_{A}$ to $\mathbf{x}_{r}$. When this happens, a point on the border of $\mathcal{F}_{A}$ is determined using a dichotomic search and a new chart is defined on it. Since the validity areas of the charts are not null in all directions and the chart is defined at the border of $\mathcal{F}_{A}$, this new chart will parametrize an area not previously in $\mathcal{F}_{A}$ and, thus $\mu\left(\mathcal{F}_{A}\right)$ is increased. Note that $\mu\left(\mathcal{F}_{A}\right)$ is increased even if this chart is close to the border of $\mathcal{F}$ and, thus, it also parametrizes regions in collision, i.e., not in $\mathcal{F}$. In practice, new charts are defined on $\mathbf{x}_{n}$ and the dichotomic search is only performed if $\mathbf{x}_{n}$ is already the center of a chart. This procedure is not detailed in Algorithm 3 for the sake of clarity. The creation of a new chart removes part of the sampling area for the chart previously parametrizing $\mathbf{x}_{n}$ and, thus, all charts will eventually become closed, i.e., charts where $\mathcal{S}_{i}=\mathcal{V}_{i}$.

Closed charts might include areas where their parametrizations are not valid, i.e., where Eqs. (7) to (9) do not hold. Under the taken assumptions, these areas have non-null measure and, thus, there is a non-null probability of generating a sample on them. When such sample is generated, the same reasoning as that used with open charts leads to an increment of $\mu\left(\mathcal{F}_{A}\right)$.

Special care must be taken to ensure that the addition of a new chart to $A$ does not decrease $\mu\left(\mathcal{F}_{A}\right)$. According to [38] in very curved regions unmapped gaps between charts might appear. The procedure at the end of the sampling process (lines 5 to 8 in Algorithm 2) allows removing these gaps, if any, ensuring that no part of $\mathcal{F}_{A}$ is lost.

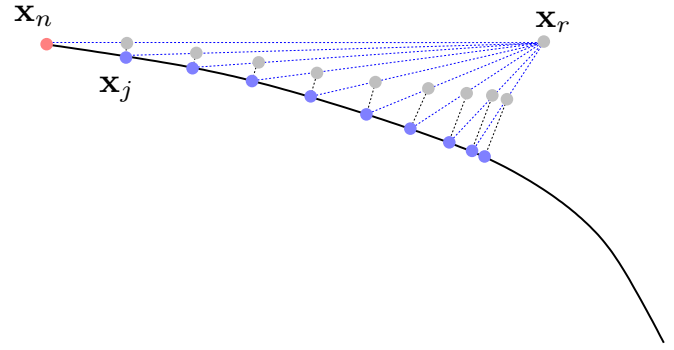

Fig. 10. The branch generation procedure used in the CB-RRT algorithm. The next sample in the RRT branch, $\mathbf{x}_{j}$, is determined by first interpolating a point in ambient space between the last point in the branch, $\mathbf{x}_{n}$, and the random sample, $\mathbf{x}_{r}$, and then projecting it on the manifold using a Jacobian pseudo-inverse procedure.

Summarizing, since $\mu(\mathcal{F})$ is finite and $\mathcal{F}_{A}$ monotonically increases with every new chart, the atlas will eventually cover $\mathcal{F}$. The extension of the atlas can not be stalled before covering $\mathcal{F}$ since, this would imply to have a chart in $A$ including a region not in $\mathcal{F}_{A}$ with null probability of being sampled, which contradicts the taken hypothesis. When the atlas is fully defined, $\mathcal{F}$ can be densely sampled and this guarantees the probabilistic completeness of the algorithm. In practice, though, paths are typically found far before the full atlas is defined.

\section{EXPERIMENTS}

We implemented the AtlasRRT planner described through Sections IV and V in C. The implementation uses SOLID [49] as collision detector, the GNU Scientific Library [50] for the linear algebra operations, and the kd-tree described in [51] for the nearest-neighbor queries. The resulting software package as well as the examples used in the experiments reported below can be downloaded from [52]. The preliminary version of AtlasRRT [27], relied on a formulation of the mechanisms with redundant variables that yields a system of simple equations only containing linear, bilinear, and quadratic monomials [53]. In this paper, we propose to use a standard Denavit-Hartenberg formulation for the joints [54]. Due to the high non-linearity of this formulation, the manifold tends to be more convoluted, which may hinder the projection of samples from the tangent to the configuration space, and the trace of this space using continuation-based methods. The experimental results show that this effect is compensated by the speed up obtained due to the reduction in the dimensionality of the ambient space.

The experiments presented herein aim to verify that the proposed planner is able to explore the configuration manifolds arising in realistic problems, even when the solution paths must traverse narrow corridors and avoid local minima. Moreover, we aim to test the sensitivity of the approach to the variations of the different parameters and its scalability with respect to the dimensionality of the configuration space. Finally, we aim to determine the efficiency of the approach, as compared with the most relevant existing methods.

For the sake of comparison we use the HC-planner [25] and CB-RRT, a planner that includes the mechanisms for path 

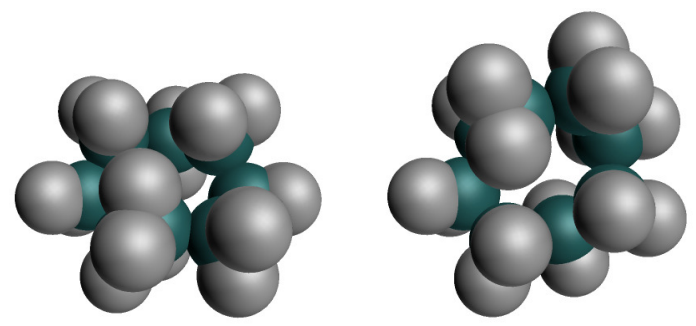

(1) Cyclooctane

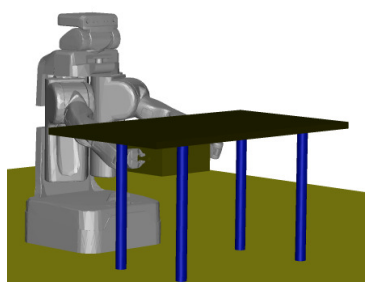

(3) PR2 with a box
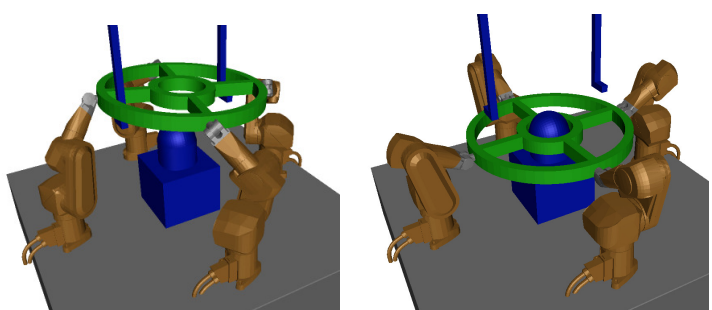

(5) Four Rx60

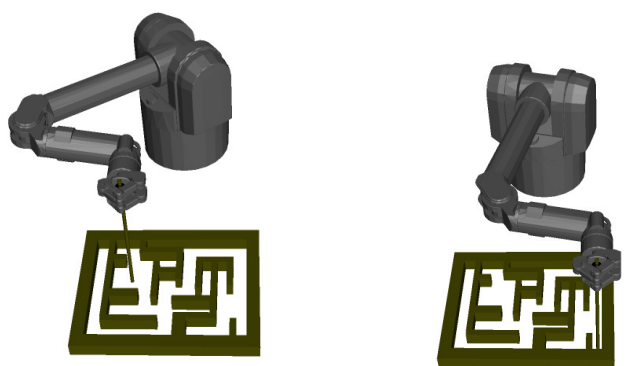

(2) Barrett arm
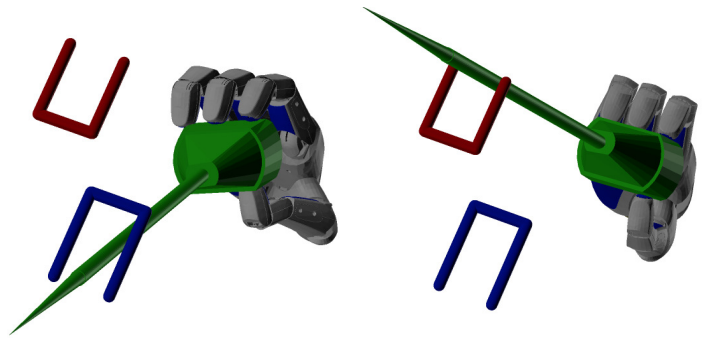

(4) Schunk hand

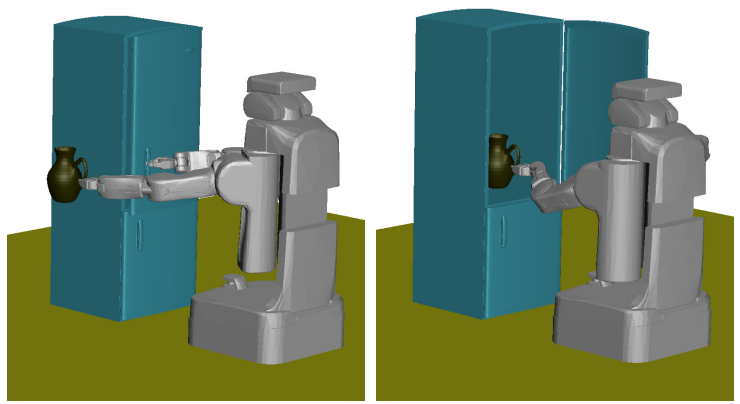

(6) PR2 with a pitcher

Fig. 11. The six benchmarks used in this paper. For each benchmark, the left and right pictures correspond to start and goal configurations, respectively. (1) The cyclooctane molecule. (2) The Barrett arm solving a maze problem. (3) The PR2 robot moving a box. (4) The Schunk anthropomorphic grasping a needle. (5) Four Säubli Rx60 arms collaborating to move a circular piece in an industrial environment. (6) The PR2 robot putting a pitcher into a fridge.

planning on manifolds of the CBiRRT2 planner introduced in [24]. The task-space aspects of CBiRRT2, however, are not included in CB-RRT since AtlasRRT does not use them. The HC-planner is a resolution complete planner on manifolds based on a greedy best first search method on a graph implicitly defined on the atlas that is build along with the search. The CB-RRT planner shares the bi-directional search strategy with AtlasRRT, but the random samples $\mathbf{x}_{r}$ are generated in the joint ambient space. Then, the algorithm interpolates between the nearest point already in the RRT, $\mathbf{x}_{n}$, and $\mathbf{x}_{r}$ to get a point $\mathbf{x}_{j}$ that is projected on the manifold using the Jacobian pseudo-inverse procedure [55], as illustrated in Fig. 10. The process of interpolation and projection is repeated to grow branches on the configuration manifold. Note, however, that this process tends to produce short branch extensions when the interpolation direction approaches the orthogonal to the manifold [35]. For a fair comparison, both the HC-planner and the CB-RRT are applied on the same formulation used by AtlasRRT.

Figure 11 shows the start and goal configurations for the six benchmarks used in this paper, sorted by increasing dimen- sionality of its configuration space. These benchmarks have been inspired by examples previously used in path planning under kinematic constraints [10, 24]. The first one is the cyclooctane, a molecule whose kinematics is a 8-revolute loop. Here, we have to find a path between two conformations that avoids collisions between carbon and hydrogen atoms (depicted in the figure in cyan and white respectively). This is a very constrained problem where the solution path requires to pass through three narrow passages. The second benchmark involves the Barrett arm solving a maze problem. The stick moved by the arm has to stay in contact with the maze plane and perpendicular to it, without rotating about its axis. In the third problem, a PR2 robot with fixed base must move a box located under a table onto this table, without rotating it. The gap between the robot and the table is narrow considering the size of the box, which increases the complexity of the problem. In the fourth problem, the Schunk anthropomorphic hand [18] grasps a needle which must be moved avoiding a couple of U-shaped obstacles that introduce local minima in the planning. In the fifth problem, four Stäubli Rx60 industrial arms must perform complex coordinated motions to extract a 
TABLE I

DIMENSION OF THE CONFIGURATION AND AMBIENT SPACES, SUCCESS RATES, NUMBER OF NODES/CHARTS, AND EXECUTION TIMES IN SECONDS FOR THE THREE METHODS COMPARED IN THIS PAPER.

\begin{tabular}{l|cc|ccc|ccc|cccc}
\hline & \multicolumn{4}{c}{ HC-Planner } & \multicolumn{3}{c}{ CB-RRT } & \multicolumn{3}{c}{ AtlasRRT } \\
\hline Benchmark & $\mathrm{k}$ & $\mathrm{n}$ & Succ. & Charts & Time & Succ. & Nodes & Time & Succ. & Charts & Nodes & Time \\
\hline (1) Cyclooctane & 2 & 8 & 0.28 & 420 & 19.67 & 1.0 & 43333 & 13.88 & 1.0 & 139 & 5812 & 1.94 \\
(2) Barrett Arm & 3 & 9 & 0.20 & 336 & 2.59 & 1.0 & 49248 & 30.23 & 1.0 & 80 & 5316 & 2.43 \\
(3) PR2 box & 4 & 16 & 0.97 & 671 & 135.36 & 1.0 & 13657 & 15.00 & 1.0 & 58 & 1393 & 0.88 \\
(4) Robot Hand & 5 & 23 & 0.25 & 1657 & 204.13 & 0.5 & 55098 & 256.22 & 1.0 & 50 & 1503 & 8.23 \\
(5) Four Rx60 & 6 & 24 & 0.01 & 1355 & 170.16 & 1.0 & 25013 & 62.87 & 1.0 & 566 & 10838 & 15.54 \\
(6) PR2 pitcher & 8 & 16 & 0.01 & 284 & 493.99 & 1.0 & 8237 & 12.31 & 1.0 & 111 & 2683 & 2.37 \\
\hline
\end{tabular}

large piece from two hooks in the ceiling and insert it into a peg. Finally, in the last problem, the PR2 robot with a fixed base has to put a pitcher into a fridge maintaining it vertical with its left arm and at the same time open the door with its right arm.

Table I shows the performance comparison between the HC-planner, the CB-RRT and the AtlasRRT, averaged over 100 runs and for a maximal execution time of 600 seconds on a Intel Core i7 at $2.93 \mathrm{Ghz}$ running Mac OS X with parameters set to $\epsilon=0.1, \alpha=0.45 \mathrm{rad}, \rho=1, \delta=0.05$, and $\lambda=2$ for all the experiments. Two different sampling radius $\rho_{s}$ are used taking into account that a small $\rho_{s}$ is more adequate in low dimensional spaces or when the problem is very constrained by obstacles and a large $\rho_{s}$ is better suited when a large configuration space must be explored. Thus, we set $\rho_{s}=2$ for experiments (1), (2), and (4) and $\rho_{s}=7$ for experiments (3), (5), and (6). For each benchmark, the table gives the dimensionality of both the configuration space, $k$, and the ambient space, $n$. It also provides for each planner, the percentage of successful runs (in the Succ. column), and for these runs, the number of charts and RRT nodes required (given in the Charts and Nodes columns, respectively), as well as the execution times in seconds (in the Time column).

A first remarkable result is that in problems such as the Barrett arm problem, where the configuration space is of low dimensionality (2-3), the HC-Planner can be even faster than the CB-RRT because the search relies on an atlas that captures the structure of the configuration manifold. However, the HC-Planner is only successful in few of the experiments because it tends to be blocked in narrow passages whereas the CB-RRT can successfully negotiate them. AtlasRRT combines the advantage of the two methods since it takes advantage of the atlas while avoiding being blocked by obstacles. In problems of moderate dimensionality (4-5), the performance of HC-Planner starts to decrease since its computational cost is exponential with the dimension of the space and CB-RRT starts to be more efficient. In these situations AtlasRRT has a better performance than the two other methods. A significant case is the Robot hand, where both HC-Planner and CB-RRT have many failures and they are more than 25 times slower than AtlasRRT, when they succeed. In higher dimension (6-8), HC-Planner is almost unable to find a solution path, whereas both the CB-RRT and the AtlasRRT are successful in all cases, with AtlasRRT being more efficient.

Since CB-RRT and AtlasRRT share the same search strat- egy, the better performance of the second can be explained by the higher quality of the samples obtained from the atlas and for the more robust branch extension mechanisms, both possible thanks to the parametrization provided by the atlas. With the aim of elucidating which of these two factors is more relevant, we repeated the experiments with AtlasRRT, but sampling in ambient space. Note that line 3 of Algorithm 3 projects the samples on the tangent space associated with the nearest node in the RRT. This cancels part of the bias introduced by sampling in the ambient space. Despite this correction factor, the execution times double with respect to those obtained with the standard AtlasRRT. The exceptions are the robot hand example where the execution time is 10 times larger and the experiment with the four Rx60 robots, where the execution time hardly varies. In this case, the advantage of the AtlasRRT is mostly provided by the more efficient branch generation procedure.

The Barrett arm experiment was used to evaluate the influence of the different parameters required by AtlasRRT. We varied $\epsilon$ from 0.05 to 0.25 in steps of 0.05 and we observed that the execution time remained in between 2 and 3 seconds in all cases. The number of charts, thought, increases from 80 to 200 when $\epsilon$ is set to the lowest value. Similar results are obtained when varying $\alpha$ from 0.2 to 0.7 in steps of 0.1 radians. When varying $\rho$ from 0.25 to 1.5 in steps of 0.25 , we observed that for the lower values of the parameter the number of charts increases up to 350 and the execution time increases up to 4 seconds. This increment is due to the overhead of creating the charts. Note, however that the performance is still remarkably good. When $\lambda$ varies from 2 to 7 in steps of one unit, neither the execution time nor the number of charts change significantly. Finally, we performed a series of experiments varying $\rho_{s}$ from 2 to 6 in steps of one unit. For large values of this parameter the execution time increases up to 6 seconds since most of the branches are stopped due to collisions, which hinders the effective exploration of the RRT. This is a well known issue of sample-based path planning that is addressed by the dynamic-domain approach [47], which adapts the size of the sampling areas in different parts of the configuration space. Such technique could be incorporated into the AtlasRRT, but we leave this point as a future work.

Finally, note that using the preliminary version of the AtlasRRT, a problem involving two Stäubli Rx60 robots and no obstacles was solved in about 14 seconds in average [27]. With the improvements introduced in this paper, the same 
problem is solved in about 0.2 seconds. These improvements allow addressing significantly more complex problems, such as the four Stäubli Rx60 example.

\section{CONCLUSIONS}

In this paper, we presented the AtlasRRT algorithm, an approach that uses an atlas to efficiently explore a configuration space manifold implicitly defined by kinematic constraints. Since defining the full atlas for a given manifold is an expensive process, the AtlasRRT algorithm intertwines the construction of the atlas and the RRT: the partially constructed atlas is used to sample new configurations and to generate new branches for the RRT, and the RRT is used to determine directions of expansion for the atlas. The approach retains the exploration bias typical of RRT approaches in the sense that the tree is strongly pushed towards yet unexplored regions of the configuration space manifold. In the experiments reported in this paper, AtlasRRT is more efficient than existing state of the art approaches although this might not be the case in problems where the configuration space is relatively similar to the ambient space, i.e., non very constrained problems. The computational tools used in AtlasRRT are more complex than the ones used in existing approaches, although we provide an implementation where new benchmarks can be easily tested [52].

A fundamental aspect not considered in the present work is the presence of singularities. When reaching a singularity, control problems might appear and the forces in the robot actuators might be undetermined, possibly leading to failures in the motors. Thus, ideally, the path planner must be able to determine singularity-free paths. This is an aspect not usually addressed in the literature and we are currently working on extensions of the presented planner to determine such paths [56]. Changes in the dimensionality of the configuration manifold also represent an issue for the presented planner. If these changes occur in the transition between consecutive stages of a given task, the presented planner can be used for each one of them separately.

Several extensions to the basic AtlasRRT algorithm can be devised. In particular, it might be useful to exploit the atlas to obtain a more meaningful distance between samples than the Euclidean one, in a way similar to what is done in [48]. Additonally, we would like to integrate cost functions to focus the planning on the relevant parts of the configuration space [57] and to explore the possible extension of the proposed planner to problems with differential constraints. Finally, the solution paths found so far are jagged, as it is usually the case with sampling-based path planning methods. We are currently investigating the possible extension of the asymptotically-optimal path planners [58] to the context of path planning under kinematic constraints [46].

\section{REFERENCES}

[1] H. Choset, K. Lynch, S. Hutchinson, G. Kantor, W. Burgard, L. Kavraki, and S. Thrun, Principles of Robot Motion: Theory, Algorithms, and Implementations. MIT Press, 2005.

[2] S. M. LaValle, Planning Algorithms. New York: Cambridge University Press, 2006.
[3] L. E. Kavraki, P. Svestka, J.-C. Latombe, and M. H. Overmars, "Probabilistic roadmaps for path planning in high-dimensional configuration spaces," IEEE Transactions on Robotics and Automation, vol. 12, pp. 566-580, 1996

[4] S. M. LaValle and J. J. Kuffner, "Rapidly-exploring random trees: Progress and prospects," in Algorithmic and Computational Robotics - New Directions (WAFR2000), 2000, pp. 293-308.

[5] Kineo Computer Aided Motion web page, http://www.kineocam.com.

[6] S. M. Lavalle, "Motion planning. Part I: The essentials," IEEE Robotics and Automation Magazine, vol. 18, no. 1, pp. 79-89, 2011.

[7] J. Canny, The Complexity of Robot Motion Planing. MIT Press, 1988.

[8] L. Han and N. M. Amato, "A kinematics-based probabilistic roadmap method for closed chain systems," in Algorithmic and Computational Robotics - New Directions (WAFR2000), 2000, pp. 233-246.

[9] J. H. Yakey, S. M. LaValle, and L. E. Kavraki, "Randomized path planning for linkages with closed kinematic chains," IEEE Transactions on Robotics and Automation, vol. 17, no. 6, pp. 951-959, 2001.

[10] J. Cortés, "Motion planning algorithms for general closed-chain mechanisms," Ph.D. dissertation, Institut National Polytechnique de Toulouse, Toulouse, France, 2003

[11] T. Siméon, J. P. Laumond, J. Cortés, and A. Sahbani, "Manipulation planning with probabilistic roadmaps," International Journal of Robotics Research, vol. 23, no. 7-8, pp. 729-746, 2004.

[12] J.-P. Merlet, Parallel Robots. Kluwer, Boston, MA, 2000.

[13] C. Rosales, L. Ros, J. M. Porta, and R. Suárez, "Synthesizing grasp configurations with specified contact regions," International Journal of Robotics Research, vol. 30, no. 4, pp. 431-443, 2011.

[14] A. Rodríguez, L. Basañez, and E. Celaya, "A relational positioning methodology for robot task specification and execution," IEEE Transactions on Robotics, vol. 24, no. 3, pp. 600-611, 2008.

[15] G. Ballantyne and F. Moll, "The da Vinci telerobotic surgical system: Virtual operative field and telepresence surgery," Surgical Clinics of North America, vol. 83, no. 6, pp. 1293-1304, 2003.

[16] W. J. Wedemeyer and H. Scheraga, "Exact analytical loop closure in proteins using polynomial equations," Journal of Computational Chemistry, vol. 20, no. 8, pp. 819-844, 1999.

[17] E. Marder-Eppstein, E. Berger, T. Foote, B. Gerkey, and K. Konolige, "The office marathon: Robust navigation in an indoor office environment," in IEEE International Conference on Robotics and Automation, 2010.

[18] Schunk Anthropomorphic Hand web page, http://www.schunk.com.

[19] C. Ott, O. Eiberger, W. Friedl, B. Bauml, U. Hillenbrand, C. Borst, A. Albu-Schafer, B. Brunner, H. Hirschmuller, and G. Hirzinger, "A humanoid two-arm system for dexterous manipulation," in IEEE-RAS International Conference on Humanoid Robots, 2006, pp. 276-283.

[20] D. Berenson, S. S. Srinivasa, D. Ferguson, and J. J. Kuffner, "Manipulation planning on constraint manifolds," in IEEE International Conference on Robotics and Automation, 2009, pp. 1383-1390.

[21] S. Dalibard, A. Nakhaei, F. Lamiraux, and J.-P. Laumond, "Whole-body task planning for a humanoid robot: a way to integrate collision avoidance," in IEEE-RAS International Conference on Humanoid Robots, 2009 , pp. 355-360.

[22] I. Havoutis and S. Ramamoorthy, "Motion synthesis through randomized exploration of submanifolds of configuration spaces," in RoboCup 2009: Robot Soccer World Cup XIII. Lecture Notes in Artificial Intelligence, vol. 5949, 2009, pp. 92-103.

[23] X. Tang, S. Thomas, P. Coleman, and N. M. Amato, "Reachable distance space: Efficient sampling-based planning for spatially constrained systems," International Journal of Robotics Research, vol. 29, no. 7, pp. 916-934, 2010.

[24] D. Berenson, S. S. Srinivasa, and J. J. Kuffner, "Task space regions: A framework for pose-constrained manipulation planning," International Journal of Robotics Research, vol. 30, no. 12, pp. 1435-1460, 2011.

[25] J. M. Porta, L. Jaillet, and O. Bohigas, "Randomized path planning on manifolds based on higher-dimensional continuation," The International Journal of Robotics Research, vol. 31, no. 2, pp. 201-215, 2012.

[26] D. Hsu, J.-C. Latombe, and H. Kurniawati, "On the probabilistic foundations of probabilistic roadmap planning," vol. 25, no. 7, pp. 627 643, 2006.

[27] L. Jaillet and J. M. Porta, "Path planning with loop closure constraints using an atlas-based RRT," International Symposium on Robotics Research, 2011.

[28] L. Han and L. Rudolph, "Inverse kinematics for a serial chain with joints under distance constraints," in Robotics: Science and Systems II, 2006, pp. 177-184. 
[29] J. Cortés and T. Siméon, "Sampling-based motion planning under kinematic loop closure constraints," 6th International Workshop on the Algorithmic Foundations of Robotics, pp. 75-90, 2004.

[30] M. Gharbi, J. Cortés, and T. Siméon, "A sampling-based path planner for dual-arm manipulation," in IEEE/ASME International Conference on Advanced Intelligent Mechatronics, 2008, pp. 383-388.

[31] A. Shkolmik and R. Tedrake, "Path planning in 1000+ dimensions using a task-space Voronoi bias," in IEEE International Conference on Robotics and Automation, 2009, pp. 2892-2898.

[32] Z. Yao and K. Gupta, "Path planning with general end-effector constraints: Using task space to guide configuration space search," in IEEE/RSJ International Conference on Intelligent Robots and Systems, 2005, pp. 1875-1880.

[33] M. Stilman, "Task constrained motion planning in robot joint space," in IEEE/RSJ International Conference on Intelligent Robots and Systems, 2007, pp. 3074-3081.

[34] D. Berenson and S. S. Srinivasa, "Probabilistically complete planning with end-effector pose constraints," in IEEE International Conference on Robotics and Automation, 2010, pp. 2724-2730.

[35] C. Suh, T. T. Um, B. Kim, H. Noh, M. Kim, and F. C. Park, "Tangent space RRT: A randomized planning algorithm on constraint manifolds,' in IEEE International Conference on Robotics and Automation, 2011, pp. 4968-4973.

[36] A. Yershova and S. M. LaValle, "Motion planning for highly constrained spaces," in Robot Motion and Control. Lecture Notes on Control and Information Sciences, vol. 396, 2009, pp. 297-306.

[37] M. P. do Carmo, Differential Geometry of Curves and Surfaces. Prentice-Hall, 1976.

[38] M. E. Henderson, "Multiple parameter continuation: Computing implicitly defined k-Manifolds," International Journal of Bifurcation and Chaos, vol. 12, no. 3, pp. 451-476, 2002.

[39] - Numerical continuation methods for dynamical systems: path following and boundary value problems. Springer, 2007, ch. HigherDimensional Continuation.

[40] B. Krauskopf, H. M. Osinga, and J. Galán-Vioque, Numerical continuation methods for dynamical systems: path following and boundary value problems. Springer, 2007.

[41] B. Roth and F. Freudenstein, "Synthesis of path-generating mechanisms by numerical methods," ASME Journal of Engineering for Industry, vol. 85 , pp. 298-307, 1963.

[42] A. J. Sommese and C. W. Wampler, The Numerical Solution of Systems of Polynomials Arising in Engineering and Science. World Scientific, 2005.

[43] F.-C. Yang and E. J. Haug, "Numerical analysis of the kinematic dexterity of mechanisms," Journal of Mechanical Design, vol. 116, pp. 119-126, 1994.

[44] C. Rosales, J. M. Porta, and L. Ros, "Global optimization of robotic grasps," in Robotics: Science and Systems, 2011, pp. 289-296.

[45] W. C. Rheinboldt, "MANPACK: A set of algorithms of computations on implicitly defined manifolds," Computers and Mathematics with Applications, vol. 32, no. 12, pp. 15-28, 1996.

[46] L. Jaillet and J. M. Porta, "Asymptotically-optimal path planning on manifolds," in Robotics: Science and Systems, 2012.

[47] A. Yershova, L. Jaillet, T. Siméon, and S. M. LaValle, "Dynamic-domain RRTs: Efficient exploration by controlling the sampling domain," in IEEE International Conference on Robotics and Automation, 2005, pp. 3856-3861.

[48] R. Chaudhry and Y. Ivanov, "Fast approximate nearest neighbor methods for non-Euclidean manifolds with applications to human activity analysis in videos," in European Conference on Computer Vision, 2010, pp. 735748 .

[49] The SOLID web page, http://www.dtecta.com.

[50] M. Galassi and et al., GNU Scientific Library Reference Manual. Network Theory Ltd., 2009.

[51] A. Yershova and S. M. LaValle, "Improving motion planning algorithms by efficient nearest neighbor searching," IEEE Transactions on Robotics, vol. 23, no. 1, pp. 151-157, 2007.

[52] The Cuik project web page, http://www.iri.upc.edu/research/ webprojects/cuikweb.

[53] J. M. Porta, L. Ros, and F. Thomas, "A linear relaxation technique for the position analysis of multiloop linkages," IEEE Transactions on Robotics, vol. 25, no. 2, pp. 225-239, 2009.

[54] J. Denavit and R. Hartenberg, "A kinematic notation for lower-pair mechanisms based on matrices," Transactions of the ASME. Journal of Applied Mechanics, vol. 23, pp. 215-221, 1955.
[55] D. E. Whitney, "Resolved motion rate control of manipulators and human prostheses," IEEE Transactions on Man-Machine Systems, vol. 10 pp. 47-53, 1969.

[56] O. Bohigas, M. E. Henderson, L. Ros, and J. M. Porta, "A singularityfree path planner for closed-chain manipulators," in IEEE International Conference on Robotics and Automation, 2012, pp. 2128-2134.

[57] L. Jaillet, J. Cortés, and T. Siméon, "Sampling-based path planning on configuration-space costmaps," IEEE Transactions on Robotics, vol. 26, no. 4, pp. 635-646, 2010.

[58] S. Karaman and E. Frazzoli, "Sampling-based algorithms for optimal motion planning," International Journal of Robotics Research, vol. 30, no. 7, pp. 846-894, 2011.

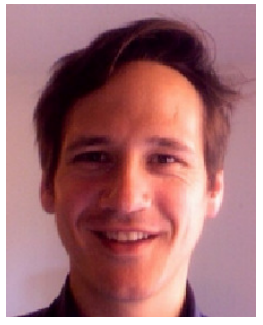

Léonard Jaillet received the Engineering degree in Mechanical Engineering from the Institut Superieur de Mecanique de Paris, Paris, France, and the Ph.D. degree in Robotics from the University of Toulouse, Toulouse, France, in 2001 and 2005, respectively. Since 2008, he has been a postdoctoral fellow at the Institut de Robòtica i Informàtica Industrial, Spanish National Research Council, Barcelona, Spain. His current research interests include motion planning for complex robotic systems and molecular simulations for structural biology.

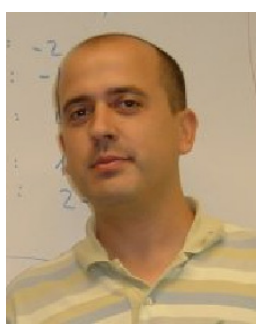

Josep M. Porta received the Engineering degree in Computer Science in 1994, and the Ph.D. degree (with honors) in Artificial Intelligence in 2001, both from the Universitat Politècnica de Catalunya, Spain. From 2001 to 2003 he held a postdoctoral position at the University of Amsterdam, pursuing research in autonomous robot localization using vision. Currently, he is an Associate Researcher of the Spanish National Research Council at the Institut de Robòtica i Informàtica Industrial (IRI, CSIC-UPC), Barcelona, Spain. His current research interests include planning under uncertainty and computational kinematics. 